with renal scarring at 1 year between the oral antibiotics group (13.7\%) and the parenteral then oral treatment group (17.7\%); subgroup analysis of the children with acute pyelonephritis detected by scintigraphy confirmed that neither treatment approach was superior. Short-term outcomes such as time to defervescence were equivalent between the treatment groups and both treatments were well tolerated.

The findings of Montini et al. indicate that it might be possible to treat children with acute pyelonephritis at home by orally administered antibiotics only; however, close follow-up by a clinician would be necessary.

Original article Montini G (2007) Antibiotic treatment for pyelonephritis in children: multicentre randomized controlled non-inferiority trial. BMJ 335: 386

\section{Patients with low-grade bladder tumors benefit from less- frequent, less-invasive follow-up}

More than half of all patients diagnosed with bladder cancer have low-grade, noninvasive papillary tumors. Current treatment comprises initial transurethral resection (TUR), sometimes with immediate chemotherapy. Patients are then followed up intensively, since this type of bladder tumor recurs frequently; however, lowgrade bladder tumors rarely progress in grade or become life threatening, so repeat surveillance every 3 months by cystoscopic examination and TUR under anesthesia might burden patients with years of frequent, uncomfortable, invasive and potentially unnecessary procedures.

Herr et al. propose that low-grade bladder cancer could be better managed using a strategy that increases the interval between cystoscopies and reduces, or even eliminates, ambulatory surgical procedures. They devised a follow-up regime involving flexible cystoscopy, a less invasive procedure, to check for recurrences every 6 months, combined with office-based fulguration (a nonsurgical treatment in which small tumors detectable by cystoscopy are destroyed using a microelectrode).

Their evaluation of 215 patients diagnosed with noninvasive papillary bladder tumors revealed that 143 patients had at least one tumor recurrence over 10 years. After a median follow-up of 8 years, there was an average of 6.2 recurrences per year, requiring 0.34 TUR per year. Only 17 patents experienced tumor progression and one patient died of bladder cancer. The authors conclude that surveillance cystoscopy every 6 months, coupled with outpatient fulguration, is sufficient to control recurrent tumors. This management strategy also reduces the therapeutic burden for patients.

Original article Herr HW et al. (2007) Management of low grade papillary bladder tumors. J Urol 178: 1201-1205

\section{Renal core biopsies distinguish malignant tumors from benign masses}

Most suspicious solid renal masses are found by radiological imaging, and are treated by radical nephrectomy; however, not all solid masses turn out to be malignant, so some operations are unnecessary for satisfactory long-term results.

The increasing sophistication of imaging techniques has enabled more accurate differentiation between benign and malignant lesions; however, interpretation can still be difficult in some cases, particularly if the tumor is smaller than $40 \mathrm{~mm}$. Lebret et al. have assessed the accuracy and safety of image-guided renal tumor biopsies in patients with smaller tumors and where radiological information is not conclusive.

They performed a total of 119 percutaneous core biopsies of renal masses over a 6 -year period. Benign lesions were diagnosed in $20.1 \%$ of biopsies and malignancy was identified in $58.8 \%$. No accurate diagnosis was possible in $21 \%$ of biopsies, but repeat biopsies in 13 patients identified a total of 11 malignant lesions. A total of 64 nephrectomies were performed, and assessment revealed that biopsy accuracy for histopathological tumor type was $86 \%$. A period of watchful waiting was proposed for 31 patients and no renal malignancies were found. CT showed stabilization or disappearance of the initial renal mass.

The authors conclude that percutaneous renal tumor biopsies are safe, cost-effective and mostly conclusive for an acute histological diagnosis. They stress that this procedure could aid accurate diagnosis and clinical decisionmaking, and could allow patients with benign lesions to avoid nephrectomy.

Original article Lebret T et al. (2007) Percutaneous core biopsy for renal masses: indications, accuracy and results. J Urol 178: 1184-1188 